\title{
A comparison of an interferon-gamma release assay and tuberculin skin test in refractory inflammatory disease patients screened for latent tuberculosis prior to the initiation of a first tumor necrosis factor $\alpha$ inhibitor
}

\author{
Arjan J. Kwakernaak • Pieternella M. Houtman • \\ Jan F. L. Weel • Johanna P. L. Spoorenberg • \\ Tim L. T. A. Jansen
}

Received: 3 February 2010 /Revised: 6 July 2010 /Accepted: 2 August 2010 / Published online: 25 August 2010

(C) The Author(s) 2010. This article is published with open access at Springerlink.com

\begin{abstract}
Treatment with TNF $\alpha$ inhibitors increases risk of reactivating a latent tuberculosislinfection (LTBI). Therefore screening, prior to therapy with $\mathrm{TNF} \alpha$ inhibitors, has been recommended, even in low-endemic areas such as well-developed Western Europe countries. We evaluated interferon-gamma release assay (IGRA), as opposed to tuberculin skin test (TST), for detection of LTBI in refractory inflammatory disease patients prior to the initiation of a first $\mathrm{TNF} \alpha$ inhibitor. In addition, we evaluated the impact of impaired cellular immunity on IGRA. Patients starting on TNF $\alpha$ inhibition were screened for LTBI by TST and IGRA (Quantiferon-TB Gold). Data on tuberculosis exposure and Bacillus Calmette-Guérin (BCG) vaccination were obtained. Cellular immunity was assessed by $\mathrm{CD}^{+} \mathrm{T}$ lymphocyte cell count. Nine out of 56 patients $(16.1 \%)$ tested positive for LTBI. A concordant positive result was present in three patients with a medical history of tuberculosis exposure. Six patients with discordant test results had either: (1) a negative TST and positive IGRA in combination with a medical history of tuberculosis exposure $(n=1)$ or (2) a positive TST and negative IGRA in
\end{abstract}

\footnotetext{
A. J. Kwakernaak • P. M. Houtman $(\bowtie) \cdot$ J. P. L. Spoorenberg

T. L. T. A. Jansen

Department of Rheumatology, Medical Center Leeuwarden, P.O. Box 888,

8901 BR Leeuwarden, The Netherlands

e-mail: n.houtman@znb.nl

J. F. L. Weel

Public Health Laboratory,

P.O.Box 21020 ,

8900 JA Leeuwarden, The Netherlands
}

combination with $\mathrm{BCG}$ vaccination $(n=3)$ or a medical history of tuberculosis exposure $(n=2)$. $\mathrm{CD}^{+} \mathrm{T}$ lymphocyte cell counts were within normal limits, and no indeterminate results of IGRA were present. IGRA appears reliable for confirming TST and excluding a false positive TST (due to prior BCG vaccination) in this Dutch serie of patients. In addition, IGRA may detect one additional case of LTBI out of 56 patients that would otherwise be missed using solely TST. Immune suppression appears not to result significantly in lower $\mathrm{CD}^{+} \mathrm{T}$ lymphocyte cell counts and indeterminate results of IGRA, despite systemic corticosteroid treatment in half of the patients. Confirmation in larger studies, including assessment of cost-effectiveness, is required.

Keywords $\mathrm{CD}^{+} \mathrm{T}$ lymphocyte cell count . IGRA - Immune-mediated inflammatory disease . Latent tuberculosis infection $\cdot \mathrm{TNF} \alpha$ inhibition $\cdot$ TST

\section{Introduction}

Tumor necrosis factor $\alpha(\mathrm{TNF} \alpha)$ is a regulating cytokine with a central role in the pathogenesis of chronic inflammatory disease and thereby a well-defined target for intervention. In concordance with this, inhibitors of TNF $\alpha$ have become increasingly important in treatment of a broad spectrum of rheumatic diseases such as rheumatoid arthritis [1], psoriatic arthritis [2], ankylosing spondylitis [3], juvenile inflammatory arthritis [4], adult onset Still's disease [5], and sarcoidosis [6]. However, TNF $\alpha$ is also 
an essential component of host defense against pathogenic viruses, bacteria, and fungi, and therapeutic inhibition of $\mathrm{TNF} \alpha$ may elicit risk of opportunistic infections [7,8], in particular, tuberculosis $[9,10]$. Thus, screening for LTBI before $\mathrm{TNF} \alpha$ inhibition has been recommended, however, no gold standard for detecting LTBI exists today and guidelines have provided conflicting recommendations about the place of diagnostic screening tests such as tuberculin skin test (TST) and interferon-gamma release assay (IGRA).

TST has several limitations as a diagnostic test in detecting LTBI. Firstly, TST attempts to measure cell-mediated immunity by delayed-type hypersensitivity response to purified protein derivate (PPD) - i.e., a crude mixture of mycobacteria antigens. This results in false positive results in nontuberculosis mycobacterium infection and clinically more important, in Bacillus Calmette-Guérin (BCG)-vaccinated persons [11, 12]. Secondly, TST sensitivity is lower in immunocompromised patients, possibly due to impaired $\mathrm{T}$ cell function and impaired cellular immunity [13]. And thirdly, TST has practical disadvantages such as inconvenience (two patient visits) and interobserver variability [14]. With respect to these limitations, an in vitro $\mathrm{T}$ cell-based assay has been developed, detecting interferon-gamma in response to contact with antigens highly specific for tuberculosis mycobacteria (ESAT-6, CFP-10, and TB 7.7). This IGRA is not influenced by contact with nontuberculosis mycobacteria or prior vaccination with $\mathrm{BCG}$ $[15,16]$. Moreover, it is suggested that IGRA has higher sensitivity in comparison to TST in patients receiving immunosuppressive treatment $[13,17,18]$. In summary, although some evidence exists that IGRA has a better performance in screening of LTBI before starting TNF $\alpha$ inhibition, the true value of IGRA as a diagnostic tool, with respect to TST, is ill-defined.

The objective of this study was to compare TST and IGRA (Quantiferon-TB Gold) in detecting LTBI in refractory inflammatory disease patients prior to the initiation of a first TNF $\alpha$ inhibitor. In addition, we evaluated the impact of cellular immunity on IGRA.

\section{Materials and methods}

Between 2008 and 2009, we prospectively enrolled patients with chronic immune-mediated inflammatory diseases starting on TNF $\alpha$ inhibition. Patients were recruited from the rheumatology outpatient clinic of the Medical Center of Leeuwarden, The Netherlands. Patients with rheumatoid arthritis, ankylosing spondylitis, psoriatic arthritis, and juvenile idiopathic arthritis (fulfilling American College of Rheumatology criteria), and two patients with sarcoidosis and Still's disease refractory to treatment with cortico- steroids and methotrexate, were included. The decision to start TNF $\alpha$ inhibition was made in agreement with criteria of the Dutch Society of Rheumatology [19]. The following variables were collected: gender, age, diagnosis, disease duration, and immunosuppressive treatment (type and dosage) at study inclusion. Tuberculosis exposure was assessed by personal and family medical history, previous treatment with tuberculostatic, and BCG vaccination. Chest radiographs were negative for (active) tuberculosis infection in all patients. Patients were tested for LTBI by TST and IGRA (Quantiferon-TB Gold). TNF $\alpha$ inhibition was initiated after a concordant negative TST and IGRA. Patients with discordant or concordant positive test results received adequate tuberculostatic treatment before initiation of TNF $\alpha$ inhibition except for BCG-vaccinated patients with positive TST, negative IGRA, and negative medical history.

TST was performed by intradermal injection of $0.1 \mathrm{ml}$ of PPD at the flexor surface of the upper one third of the forearm according to the Mantoux method. The diameter of skin induration was assessed 48-72 $\mathrm{h}$ after inoculation by a trained rheumatologic nurse. A positive TST was defined as $\geq 5 \mathrm{~mm}$ induration according to the Dutch guidelines [19]. Immediately after the intradermal injection of PPD, a heparinized whole blood sample was collected and transferred to three Quantiferon ${ }^{\mathrm{R}}$-TB gold test tubes (Cellestis Ltd, Australia) to prohibit a booster effect. IGRA was performed and interpreted according to instructions of the manufacturer and guideline of Centers of Disease Control and Prevention [20], respectively. An EDTA tube was collected for assessment of cellular immunity by $\mathrm{CD} 4^{+}$cell $\mathrm{T}$ lymphocyte count (CD4 ${ }^{+}$cell count).

\section{Results}

Patient characteristics Twenty-nine out of 56 included patients were diagnosed with rheumatoid arthritis (51.8\%) of whom $83.3 \%$ were rheumatoid factor positive (Table 1). Other diagnoses were ankylosing spondylitis (30.4\%), psoriatic arthritis $(8.9 \%)$, undifferentiated spondylarthropathy (3.6\%), juvenile idiopathic arthritis (1.8\%), adult onset Still's disease (1.8\%), and sarcoidosis (1.8\%). Mean ( \pm SD) age of the total study population was $50.3 \pm 15.4$ years, and disease duration was $8.7 \pm 9.2$ years. About half of the study population $(48.2 \%)$ had received methotrexate ( \pm corticosteroids), 5.4\% leflunomide ( \pm methotrexate \pm corticosteroids), $1.8 \%$ azathioprine and corticosteroids, $5 \%$ only corticosteroids, and $35.7 \%$ did not had previous immunosuppressive treatment. Dosage of immunosuppressive treatment is listed in Table 1. The incidence of BCG vaccination in the total investigated population was $5 \%$ which is representative of a post-World War II population. Tubercu- 
Table 1 Patient characteristics of 56 patients

\begin{tabular}{|c|c|}
\hline Patient characteristics & No. $(\%)$ \\
\hline Male & $32(53.6 \%)$ \\
\hline Age $($ mean \pm SD) & $50.3 \pm 15.4$ years \\
\hline \multicolumn{2}{|l|}{ Diagnosis } \\
\hline Rheumatoid arthritis ${ }^{\mathrm{a}}$ & $29(51.8 \%)$ \\
\hline RF positive & $25(83.3 \%)$ \\
\hline Ankylosing spondylitis ${ }^{\mathrm{a}}$ & $17(30.4 \%)$ \\
\hline Psoriatic arthritis ${ }^{\mathrm{a}}$ & $5(8.9 \%)$ \\
\hline Undifferentiated spondylitis & $2(3.6 \%)$ \\
\hline Juvenile idiopathic arthritis ${ }^{a}$ & $1(1.8 \%)$ \\
\hline Still's disease & $1(1.8 \%)$ \\
\hline Sarcoidosis & $1(1.8 \%)$ \\
\hline \multicolumn{2}{|l|}{ Disease duration (mean $\pm \mathrm{SD})$} \\
\hline Total study population & $8.7 \pm 9.2$ years \\
\hline Rheumatoid arthritis & $7.4 \pm 7.7$ years \\
\hline \multicolumn{2}{|l|}{ Type of immunosuppressive treatment (at study inclusion) } \\
\hline Methotrexate \pm corticosteroids & $27(48.2 \%)$ \\
\hline Leflunomide \pm methotrexate \pm corticosteroids & $3(5.4 \%)$ \\
\hline Azathioprine and corticosteroids & $1(1.8 \%)$ \\
\hline Only corticosteroids (maintenance and high-dose treatment) & $5(8.9 \%)$ \\
\hline No current immunosuppressive treatment & $20(35.7 \%)$ \\
\hline \multicolumn{2}{|c|}{ Daily dose of immunosuppressive treatment (at study inclusion; mean $\pm \mathrm{SD}$ ) } \\
\hline Methotrexate & $19 \pm 7 \mathrm{mg}$ \\
\hline Leflunomide & $20 \pm 0 \mathrm{mg}$ \\
\hline Azathioprine & $150 \mathrm{mg}$ \\
\hline \multicolumn{2}{|l|}{ Corticosteroids } \\
\hline Maintenance treatment & $6 \pm 2 \mathrm{mg}$ \\
\hline High-dose treatment ${ }^{\mathrm{b}}$ & $3,000 \pm 0 \mathrm{mg}$ \\
\hline BCG vaccination & $3(5 \%)$ \\
\hline Tuberculosis exposure & $8(14 \%)$ \\
\hline
\end{tabular}

Data are presented as number (No.) and percentage (\%) and, if applicable, mean \pm standard deviation (SD)

No. number of patients, $R F$ rheumatoid factor

${ }^{a}$ Diagnosis according to the American College of Rheumatology criteria

${ }^{\mathrm{b}}$ Once-only three pulses of $1 \mathrm{~g}$ methylprednisolone prior to LTBI screening $(n=3)$ losis exposure was $14 \%$ and is in line with the low prevalence of tuberculosis in Western Europe countries. The absence of reactivation of tuberculosis during $\mathrm{TNF} \alpha$ inhibition in the BCG-vaccinated patients ruled out the coexistence of BCG vaccination and prior tuberculosis exposure in this population.

Comparison of TST and IGRA Nine out of 56 patients (16.1\%) tested positive for LTBI with either TST or IGRA (Table 2). A concordant positive result was present in three patients with a medical history of tuberculosis exposure. In six patients, discordant results were obtained: one patient had a negative TST and positive IGRA, and five patients had a positive TST and negative IGRA. The mother of the patient with negative TST and positive IGRA had suffered from tuberculosis during his childhood. The five patients with positive TST and negative IGRA were either BCG-vaccinated (three patients) or had a medical history of tuberculosis exposure (two patients). The BCG-vaccinated patients with positive TST and negative IGRA did not develop tuberculosis during TNF $\alpha$ inhibition in the follow-up period (1-2 years). Interestingly, there were no indeterminate results of IGRA.

Cellular immunity $\mathrm{CD}^{+}$cell counts were within normal limits with a mean $\left( \pm\right.$ SD) of $964 \pm 568$ cells $/ \mathrm{mm}^{3}$ (Fig. 1). $\mathrm{A} \mathrm{CD}^{+}$cell count below 300 cells $/ \mathrm{mm}^{3}$ was present in two patients receiving methotrexate and low-dose corticosteroids. These two patients had a negative result for TST and IGRA and did not develop tuberculosis during TNF $\alpha$ inhibition ( 2 years of follow-up). There were no patients with a $\mathrm{CD}^{+}$cell count below 200 cells $/ \mathrm{mm}^{3}$.

\section{Discussion}

The objective of this study was to compare TST and IGRA (Quantiferon-TB Gold) in detecting LTBI in refractory 
Table 2 Characteristics of nine patients with concordant positive TST and IGRA and discordant TST and IGRA

\begin{tabular}{|c|c|c|c|c|c|c|c|}
\hline $\mathrm{N}$ & TST & IGRA & BCG & Tuberculosis exposure & $\mathrm{CD}^{+}$count $\left(\right.$cells $\left./ \mathrm{mm}^{3}\right)$ & Medication (daily dose in mg) & Diagnosis \\
\hline \multicolumn{8}{|c|}{ Concordant positive results of TST and IGRA } \\
\hline 1 & pos. & pos. & no & yes & 748 & MTX 12.5; lef 20 & PsA \\
\hline 2 & pos. & pos. & no & yes & 972 & MTX 25 & RA \\
\hline 3 & pos. & pos. & no & yes & N.A. & none & AS \\
\hline \multicolumn{8}{|c|}{ Discordant results of TST and IGRA } \\
\hline 1 & neg. & pos. & no & yes & 974 & MTX 15 & RA \\
\hline 2 & pos. & neg. & yes & no & 590 & pred 5; MTX 10; lef 20 & RA \\
\hline 3 & pos. & neg. & yes & no & 387 & MTX 25 & RA \\
\hline 4 & pos. & neg. & yes & no & 2,050 & MTX 7.5 & PsA \\
\hline 5 & pos. & neg. & no & yes & 632 & none & AS \\
\hline 6 & pos. & neg. & no & yes & 549 & MTX 7.5 & RA \\
\hline
\end{tabular}

$N$ patient number, TST tuberculosis skin test, IGRA interferon-gamma release assay (Quantiferon-TB Gold), BCG Bacillus Calmette-Guérin, $T B$ tuberculosis, $C D 4^{+}$count $\mathrm{CD}^{+}$T lymphocyte cell count, pos. positive, neg. negative, N.A. not available, MTX methotrexate, pred prednisone, lef leflunomide, $P S A$ psoriatic arthritis, $R A$ rheumatoid arthritis, $A S$ ankylosing spondylitis

inflammatory disease patients prior to the initiation of a first $\mathrm{TNF} \alpha$ inhibitor. A well-recognized problem in screening for LTBI is absence of a gold standard and thereby sensitivity and specificity of TST and IGRA cannot be directly measured. Nevertheless, assessment of tuberculosis exposure, combined with results of TST and IGRA, may roughly estimate the a priori chance of LTBI.

Nine out of 56 patients (16.1\%) tested positive for LTBI with either TST or IGRA. A concordant positive result was
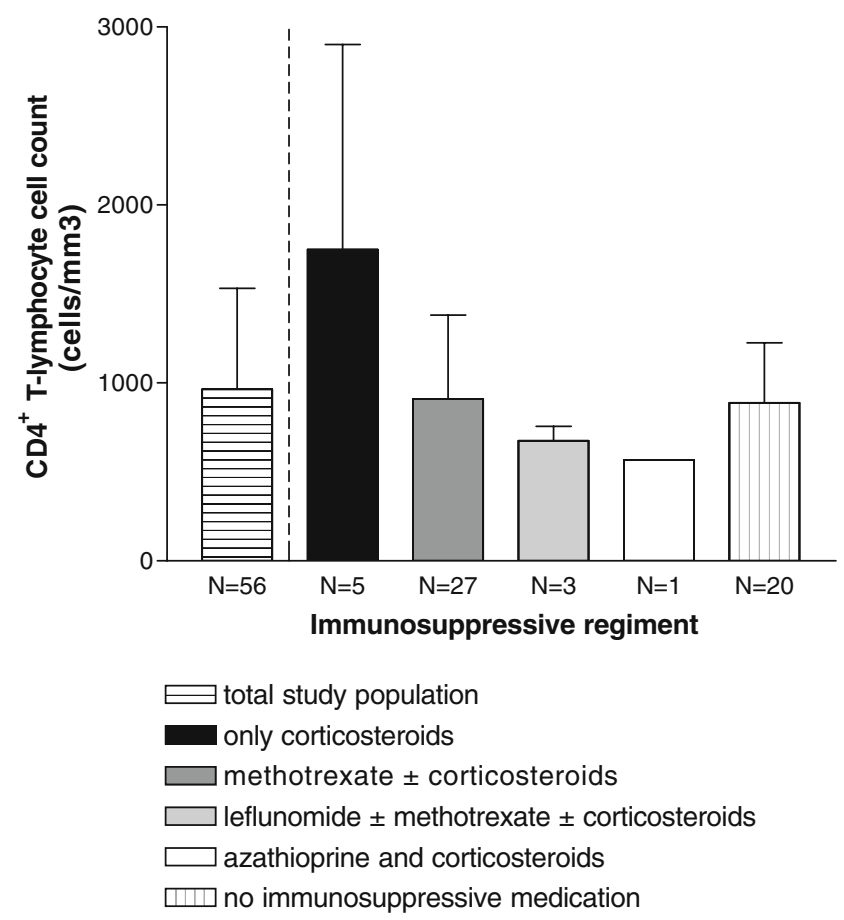

Fig. 1 Cellular immunity defined by immunosuppressive treatment and $\mathrm{CD}^{+} \mathrm{T}$ lymphocyte cell count $\left(\right.$ cells $\left./ \mathrm{mm}^{3}\right)$ at study inclusion. Data are given as mean \pm standard deviation (SD) present in three patients with a medical history of tuberculosis exposure. The remaining six patients with discordance had either a negative TST and positive IGRA (one patient) or a positive TST and negative IGRA (five patients). The discordance in the five patients with positive TST and negative IGRA can be attributed to BCG vaccination (three patients) or a medical history of tuberculosis exposure (two patients). As for the patients with negative IGRA and positive TST, it cannot be excluded that IGRA may be false negative as the infection occurred in the distant past. This may be explained by the fact that IGRA mostly measures effector $\mathrm{T}$ cell responses whereas TST measures both effector and memory $\mathrm{T}$ cell responses. After $24 \mathrm{~h}$ incubation in the IGRA, only circulating effector memory $\mathrm{T}$ cells have sufficient time to produce interferon, while central memory $\mathrm{T}$ cells first started producing interferon after a more prolonged $(72 \mathrm{~h}$ in TST) incubation [21]. The Quantiferon-TB Gold performs well in routine screening of low-prevalence populations, but its performance turned out to be suboptimal in healthy persons with a high risk of tuberculosis exposure [22]. It is also known that the sensitivity of the Quantiferon depends on the test and is higher for the latest in-tube version that was used in this study [23]. Indeed, we found a low number $(n=1)$ of discordant negative TST and positive IGRA in a low tuberculosis-exposed population.

Indeterminate results of IGRA are commonly reported in patients with, e.g., HIV, malignancy, and chronic renal failure, and patients undergoing immunosuppressive treatment $[24,25]$. In comparison with studies in rheumatic disease patients [26-32], it is remarkable that in this study IGRA could be interpreted without problems-i.e., there were no indeterminate results. This suggests that lymphocytes retained the capacity to produce interferon-gamma on 
mitogen stimulation in vitro, even with immunosuppressive treatment. Although we do not have a representative control group, it appears that $\mathrm{CD}^{+}$cell count in this study population was not greatly reduced in response to immunosuppressive treatment-i.e., $\mathrm{CD}^{+}$cell counts were not below 200 cells $/ \mathrm{mm}^{3}$ and only two patients had a $\mathrm{CD}^{+}$cell count below 300 cells $/ \mathrm{mm}^{3}$. Furthermore, the two patients with a positive TST, a negative IGRA and an positive medical history of LTBI had both a normal $\mathrm{CD}^{+}$cell count.

The absence of a significant influence of immunosuppressive treatment on interpretation of IGRA in patients with inflammatory rheumatic conditions has previously been reported by Matulis [27]. Cellestis stated that as long as the viable $\mathrm{CD}^{+}$cell count is above 200 cells $/ \mathrm{mm}^{3}$ Quantiferon-TB Gold has a good performance. However, it has been questioned whether $\mathrm{CD}^{+}$cell count can be used as a marker of validity of IGRA in patients other than HIV patients [28]. Furthermore, the intrinsic function of $\mathrm{T}$ cells may be an important precondition for the capacity of interferon production. Two studies analyzing the impact of different classes of drugs on the response of TST and IGRA in European patients with immune-mediated inflammatory diseases stated that corticosteroid treatment can in fact compromise test results by increasing the number of indeterminate results of IGRA and negative TST outcomes $[27,29]$. We therefore hypothesize that high-dose corticosteroid maintenance therapy may be a risk factor for an indeterminate IGRA result. Possibly, the absence of indeterminate test IGRA results in our study was due to the lowdose corticosteroids (10 mg per day at most). Overall, sensitivity of IGRA for the diagnosis of LTBI might be higher than TST, despite a higher rate of indeterminate results in immunocompromised patients [26-32].

Various recommendations and guidelines have been issued to determine the place of diagnostic screening test for LTBI. They are traditionally based on medical history, chest X-ray and TST. Two leading institutes, the Centers of Disease Control and Prevention (CDC) of the United States and United Kingdom's National Institute for health and Clinical Excellence (NICE), have developed guidelines regarding correct usage of both diagnostic tools [20, 33], but they came to conflicting conclusions. The CDC stated that IGRA is a real alternative for TST whereas NICE advised the use of IGRA only for the validation of a positive TST. The variation in prevalence of tuberculosis over time in different parts of the world may account for this disagreement. Western Europe has a substantially larger BCG-vaccinated population as compared to the United States of America (USA). Therefore, the addition of IGRA augments the specificity of prescreening in Western Europe. Furthermore, most cases of LBTI in Western Europe occurred in a population born before 1945. A lower performance of IGRA in Europe as compared to the USA may be attributed to a low sensitivity in the case of longer duration of LTBI. In different countries of Western Europe IGRA is currently added in a second step approach according to NICE or is even replacing TST (Germany, Switzerland) $[34,35]$. We feel that all physicians, including rheumatologists, who treat different immune-mediated inflammatory diseases, are obliged to hand tailor the prophylactic therapy for LTBI by the prevalence of tuberculosis and BCG vaccination of their country.

In conclusion, the findings of this study suggest that IGRA appears reliable for confirming a positive TST and excluding a false positive result (in case of prior BCG vaccination) in this Dutch serie of patients. IGRA may thereby reduce the number of patients in whom tuberculostatics are prescribed in absence of LTBI, resulting in an evident benefit of avoiding a 6-month delay of effective anti-rheumatic treatment with TNF $\alpha$ inhibition and possible side effects due to treatment with tuberculostatics. Moreover, IGRA seems to detect an additional case of LTBI that otherwise be missed using solely TST. IGRA appears not to be significantly influenced by immunosuppressive treatment, at least not by the regimen and dosage used in this study. Although the number of patients is not large enough for powerful statistical analysis and patient characteristics are quite heterogeneous, this study contributes to the ongoing discussion on the diagnostic value of IGRA. IGRA should not be employed as an alternative single screening test as false negative results have been observed. Confirmation in larger studies, including cost-effectiveness in different countries to determine the optimal screening strategy, is warranted.

\section{Disclosures None}

Open Access This article is distributed under the terms of the Creative Commons Attribution Noncommercial License which permits any noncommercial use, distribution, and reproduction in any medium, provided the original author(s) and source are credited.

\section{References}

1. Smolen JS, Aletaha D, Koeller M, Weisman MH, Emery P (2007) New therapies for treatment of rheumatoid arthritis. Lancet 370:1861-1874

2. Mease PJ (2009) Psoriatic arthritis assessment and treatment update. Curr Opin Rheumatol 21:348-355

3. Braun J, Davis J, Dougados M, Sieper J, van der Linden S, van der Heijde D (2006) First update of the international ASAS consensus statement for the use of anti-TNF agents in patients with ankylosing spondylitis. Ann Rheum Dis 65:316-320

4. Quartier P, Taupin P, Bourdeaut F et al (2003) Efficacy of etanercept for the treatment of juvenile idiopathic arthritis according to the onset type. Arthritis Rheum 48:1093-1101 
5. Kraetsch HG, Antoni C, Kalden JR, Manger B (2001) Successful treatment of a small cohort of patients with adult onset of Still's disease with infliximab: first experiences. Ann Rheum Dis 60 (Suppl 3):iii55-iii57

6. Saleh S, Ghodsian S, Yakimova V, Henderson J, Sharma OP (2006) Effectiveness of infliximab in treating selected patients with sarcoidosis. Respir Med 100:2053-2059

7. Bongartz T, Sutton AJ, Sweeting MJ, Buchan I, Matteson EL, Montori V (2006) Anti-TNF antibody therapy in rheumatoid arthritis and the risk of serious infections and malignancies: systematic review and meta-analysis of rare harmful effects in randomized controlled trials. JAMA 295:2275-2285

8. Patkar NM, Teng GG, Curtis JR, Saag KG (2008) Association of infections and tuberculosis with antitumor necrosis factor alpha therapy. Curr Opin Rheumatol 20:320-326

9. Keane J, Gershon S, Wise RP et al (2001) Tuberculosis associated with infliximab, a tumor necrosis factor alpha-neutralizing agent. N Engl J Med 345:1098-1104

10. Gomez-Reino JJ, Carmona L, Angel Descalzo M (2007) Risk of tuberculosis in patients treated with tumor necrosis factor antagonists due to incomplete prevention of reactivation of latent infection. Arthritis Rheum 57:756-761

11. Andersen P, Munk ME, Pollock JM, Doherty TM (2000) Specific immune-based diagnosis of tuberculosis. Lancet 356:1099-1104

12. Diagnostic Standards and Classification of Tuberculosis in Adults and Children. This official statement of the American Thoracic Society and the Centers for Disease Control and Prevention was adopted by the ATS Board of Directors, July 1999. This statement was endorsed by the Council of the Infectious Disease Society of America, September 1999. Am J Respir Crit Care Med 2000; 161:1376-1395

13. Chen DY, Shen GH, Hsieh TY, Hsieh CW, Lan JL (2008) Effectiveness of the combination of a whole-blood interferongamma assay and the tuberculin skin test in detecting latent tuberculosis infection in rheumatoid arthritis patients receiving adalimumab therapy. Arthritis Rheum 59:800-806

14. Pouchot J, Grasland A, Collet C, Coste J, Esdaile JM, Vinceneux P (1997) Reliability of tuberculin skin test measurement. Ann Intern Med 126:210-214

15. Brock I, Weldingh K, Lillebaek T, Follmann F, Andersen P (2004) Comparison of tuberculin skin test and new specific blood test in tuberculosis contacts. Am J Respir Crit Care Med 170:65-69

16. Kang YA, Lee HW, Yoon HI et al (2005) Discrepancy between the tuberculin skin test and the whole-blood interferon gamma assay for the diagnosis of latent tuberculosis infection in an intermediate tuberculosis-burden country. JAMA 293:2756-2761

17. Pai M, Riley LW, Colford JM Jr (2004) Interferon-gamma assays in the immunodiagnosis of tuberculosis: a systematic review. Lancet Infect Dis 4:761-776

18. Lalvani A (2007) Diagnosing tuberculosis infection in the 21st century: new tools to tackle an old enemy. Chest 131(6):1898-1906

19. Dutch Society of Rheumatology (2003) Tuberculosis and TNFablockade (guideline)

20. Mazurek GH, Villarino ME (2003) Guidelines for using the QuantiFERON-TB test for diagnosing latent mycobacterium tuberculosis infection. Centers for disease control and prevention. MMWR Recomm Rep 31:15-18
21. Leyten EM, Arend SM, Prins C, Cobelens FG, Ottenhoff TH, van Dissel JT (2007) Discrepancy between mycobacterium tuberculosisspecific gamma interferon release assays using short and prolonged in vitro incubation. Clin Vaccine Immunol 14:880-885

22. O'Neal S, Hedberg K, Markum A, Schafer S (2009) Discordant tuberculin skin and interferon-gamma tests during contact investigations: a dilemma for tuberculosis controllers. Int $\mathrm{J}$ Tuberc Lung Dis 13(5):662-664

23. Saracino A, Scotto G, Fornabaio C et al (2009) Quantiferon-TB Gold in-tube test (QFT-GIT) for the screening of latent tuberculosis in recent immigrants in Italy. New Microbiol 32:369-376

24. Kobashi Y, Obase Y, Fukuda M, Miyashita N, Oka M (2007) Clinical evaluation of QuantiFERON TB-2G test for immunocompromised patients. Eur Respir J 30:945-950

25. Richeldi L, Losi M, D'Amico R et al (2010) Performance of tests for latent tuberculosis in different groups of immunocompromised patients. Chest 136:198-204

26. Greenberg JD, Reddy SM, Schloss SG et al (2008) Comparison of an in vitro tuberculosis interferon-gamma assay with delayed-type hypersensitivity testing for detection of latent mycobacterium tuberculosis: a pilot study in rheumatoid arthritis. J Rheumatol 35:770-775

27. Matulis G, Juni P, Villiger PM, Gadola SD (2008) Detection of latent tuberculosis in immunosuppressed patients with autoimmune diseases: performance of a mycobacterium tuberculosis antigen-specific interferon gamma assay. Ann Rheum Dis 67:84-90

28. Soborg B, Ruhwald M, Hetland ML et al (2009) Comparison of screening procedures for mycobacterium tuberculosis infection among patients with inflammatory diseases. J Rheumatol 36:1876-1884, 27

29. Inanc N, Aydin SZ, Karakurt S, Atagunduz P, Yavuz S, Direskeneli H (2009) Agreement between Quantiferon-TB Gold test and tuberculin skin test in the identification of latent tuberculosis infection in patients with rheumatoid arthritis and ankylosing spondylitis. J Rheumatol 36:2675-2681

30. Bartalesi F, Vicidomini S, Goletti D et al (2009) Quantiferon-TB Gold and the TST are both useful for latent tuberculosis infection screening in autoimmune diseases. Eur Respir J 33:586-593

31. Kim EY, Jung JY, Son JY et al (2009) Performance of the tuberculin skin test and interferon-gamma release assay for detection of tuberculosis infection in immunocompromised patients in a BCGvaccinated population. BMC Infect Dis 15:9-207

32. Martin J, Walsh C, Gibbs A et al (2010) Comparison of interferon-\{gamma $\}$-release assays and conventional screening tests before tumour necrosis factor-\{alpha\} blockade in patients with inflammatory arthritis. Ann Rheum Dis 69:181-185

33. British National Institute for Health and Clinical Excellence (2006). Tuberculosis; clinical diagnosis and management of tuberculosis and measures for its prevention and control (guideline). London, UK:NICE:2006. http://www.nice.org.uk/guidance/ CG33. Accessed March 2009

34. Beglinger C, Dudler J, Mottet $C$ et al (2007) Screening for tuberculosis infection before the initiation of an anti-TNF-alpha therapy. Swiss Med Wkly 137:620-622

35. Diel R, Hauer B, Loddenkemper R, Manger B, Kruger K (2009) Recommendations for tuberculosis screening before initiation of TNF-alpha-inhibitor treatment in rheumatic diseases. Pneumologie 63:329-334 